\title{
Yes we can analyse amyloid images - Now What?
}

\author{
Henryk Barthel ${ }^{1}$ - John Seibyl ${ }^{2}$ - Osama Sabri ${ }^{1}$
}

Published online: 9 February 2017

(C) Springer-Verlag Berlin Heidelberg 2017

With the recent introduction of amyloid PET tracers into clinical use, non-invasive biomarker information on brain amyloidosis is available which challenges the long-accepted gold standard of post mortem histopathology for diagnosing Alzheimer's disease (AD). Phase 3 amyloid PET studies in end of life patients with post mortem verification of brain amyloid status have arguably shifted the time-point of diagnosing amyloid pathology to ante mortem. Florbetapir, florbetaben, and flutemetamol, the PET tracers utilised for that purpose, share high sensitivity/specificity in detecting/ excluding beta-amyloid aggregates which accumulate in the neocortex of AD brains [1-3]. Further, comparably high discrimination accuracy was reported for these tracers between patients with clinical diagnosis of $\mathrm{AD}$ dementia and healthy controls $[4,5]$. The main advantages of these tracers are the high image signal associated with these "hot spot" imaging tracers and the binary nature of the PET result when applied in certain scenarios (reproducible mild cognitive impairment, early-onset $\mathrm{AD}$ dementia, "possible" $\mathrm{AD}$ dementia according to clinical testing $[6,7])$. This is because, in line with the amyloid cascade theory of $\mathrm{AD}$ [8], amyloid pathology should be full-blown by the time cognitive decline manifests. This favourable scenario motivated the regulatory authorities to approve these tracers based solely on a visual PET assessment by readers who have passed a tracer-specific training.

This Editorial Commentary refers to the article http://dx.doi.org/10.1007/ s00259-016-3601-4.

Henryk Barthel

henryk.barthel@medizin.uni-leipzig.de

1 Department of Nuclear Medicine, Leipzig University, Liebigstr. 18, 04103 Leipzig, Germany

2 Institute for Neurodegenerative Disorders, New Haven, USA
Respective training programs utilised for that purpose describe a series of rules referent to the question of whether there is grey vs. white matter contrast. In parallel to these efforts to establish read algorithms, in the USA the IDEAS study [9] is underway, aimed at providing outcome data about the clinical relevance of amyloid PET crucial for future reimbursement. In Europe, this effort will soon be followed by the AMYPAD study [10]. Besides the use of amyloid PET for primary diagnosis of brain amyloid pathology in conjunction with $\mathrm{AD}$, the above tracers are intensively utilized in drug testing trials [11] for confirming eligibility and monitoring the impact of therapy on brain amyloidosis.

Yet, like any diagnostic test, PET amyloid visual assessments are not perfect and technical and biological factors pose challenges to accurate scan interpretation, particularly for the inexperienced reader. Further, some concerns have been raised in the past on the validity of visual read rules [12] which might result in relevant inter-reader variability [13]. One principle advantage of the PET imaging technique lies in its quantitative nature. The question of how important quantitative data are in cases of amyloid PET image analysis depends on the question to be addressed by the PET procedure. There is no doubt that in cases where amyloid imaging is used for follow-up evaluation/therapy monitoring quantitation is important. In this instance quantitative methods using dynamic imaging and kinetic modelling should ideally be performed. However, up to now, the logistical practicalities involved in large multi-centre trials favour the simpler semiquantitative, albeit biased standardized uptake value ratio (SUVr) approach. Further, with the prospect of effective and safe amyloid aggregate-cleaving drugs comes the requirement of early and accurate identification of pathologic brain amyloid, including in subjects at high risk, but prior to cognitive symptoms. In such scenarios where there might be less pronounced amyloid load, quantitative measures might prove more 
sensitive than the standard visual interpretation. Even for the current clinical use situation (above appropriate use criteria, binary diagnosis, no anti-amyloid treatment option), however, the value of amyloid PET quantitation as an adjunct (or potential substitute) to a visual read has so far not been sufficiently investigated.

In this issue of The European Journal of Nuclear Medicine and Molecular Imaging, Pontecorvo et al. present interesting data in this context [14]. Their paper describes results of a study which investigated whether it is possible to implement quantitation into the visual florbetapir amyloid PET data interpretation, and whether this improves the visual read across readers of differing ability. For that purpose, not less than 80 readers were each asked to visually evaluate close to 100 PET data (histopathology and clinical utility trials) on day 1 , followed by a combined visual read/quantitative analysis of the same data on day 2 . As relative tracer uptake quantitation readout, static 50-60 min p.i. regional and global SUVrs taking the whole cerebellum as reference region were determined. This was accomplished, in different reader cohorts, by three different amyloid image analysis tools (MIMneuro®, Siemens syngo.PET Amyloid plaque, and Hermes BRASS ${ }^{\mathrm{TM}}$ ). The obtained SUVrs were taken as an adjunct to the visual evaluation according to a pre-defined multi-step procedure. The readers were categorized according to their accuracy achieved when analysing phase 3 histopathology cases, and the "below average" and "above average" reader data were included in this analysis. It was found that with similar degree for the three quantification tools - accuracy of visual analysis improved by taking SUVrs into consideration. This effect was more pronounced in "below average" as compared to "above average" readers.

The results presented in this paper convincingly add to our current knowledge on the reader training tools being effective in teaching physicians how to visually analyse amyloid images $[15,16]$. The reported gain of visual read accuracy by taking SUVrs into consideration is also in accordance with the fact that visual read and SUVr results might differ within at least some amyloid PET cases [17-19]. Also in accordance with the Pontecorvo et al. data, it was recently shown that taking SUVrs into account decreases inter-reader variability of amyloid PET diagnosis [20]. It would be useful to better understand in the present study the discordance between visual and quantitative assessments, and in what settings this occurred. For example, spatial normalisation algorithms may have more difficulty with cortical atrophy than human readers. It would also be interesting for the Pontecorvo et al. and other studies to see whether the concept of supporting visual read by taking SUVrs into consideration works not only on a global, but also on a regional brain level.

With regard to improved accuracy and confidence of visual amyloid image read, other support apart from SUVrs [21-23] might in the future be of value, like approaches supporting the crucial grey vs. white matter segmentation of the PET signal (MRI coregistration), approaches presenting Z-score maps as obtained by comparing the subject's PET dataset with a normal database [24-26], or uptake pattern recognition tools, like histogram analyses [27, 28], spectral analyses [29], or approaches which analyse the geometrical appearance of isointensity surfaces [30]. More advanced data analysis approaches may employ support vector machines [31]. Whether this development will in the end lead to scenarios which, as predicted by some researchers for the machine learning technology [32], will challenge the necessity of medical doctors remains, for the time being, an open and provocative question. Future research on this topic should also address the issues of (i) whether it might be feasible and worthwhile to visually analyse partial volume error-corrected [33] instead of atrophy-affected uncorrected amyloid images, (ii) whether the PET scanner quality has an influence on the visual amyloid image read which would potentially require consideration in reader training, and (iii) whether amyloid image reading rules might require some specifications with regard to the different imaging time-windows in which it is possible to, at least for some of the approved amyloid tracers, acquire the PET data.

Taken together, there is growing evidence on the efficacy of the visual read combined with quantitative information carried out in the currently established clinical context. At least in some situations, the visual read benefits from the consideration of quantitation. This is despite the overall effect size achievable for improved accuracy is limited, prompting the question on whether it is worth implementing a software tool for that purpose, particularly in cases of experienced readers. As an answer, one could argue that, to firmly establish a PET-based diagnosis, especially one with potential therapeutic consequences, it is always of advantage to have concordant visual and quantitative results. However, more work is required to investigate this feature for new, potentially emerging applications for amyloid imaging in earlier AD stages or for follow-up evaluation, as well as with regard to the question of whether tools that are capable of quantifying amyloid tracer uptake might in the future have the potential to replace visual analysis.

So what now? Should a visual/quantitative method be de rigueur for all clinical PET units? Further fieldwork would be of value in understanding the bottom-line benefits, ease of implementation/training and costs to the frontline clinical nuclear medicine facilities in assessing the broad range of patients with cognitive impairment or at risk of the same. Regardless, amyloid imaging is on a successful path to complementing the portfolio of nuclear brain imaging techniques that are straightforward to perform and that result in images that are straightforward to analyse in a clinical routine scenario, an important step towards full acceptancy by the payers and referring clinical partners, all to the benefit of our patients. 


\section{References}

1. Clark CM, Schneider JA, Bedell BJ, Beach TG, Bilker WB, Mintun MA, et al. Skovronsky DM; AV45-A07 Study Group. use of florbetapir-PET for imaging beta-amyloid pathology. JAMA. 2011;305(3):275-83.

2. Sabri O, Sabbagh MN, Seibyl J, Barthel H, Akatsu H, Ouchi Y, et al. Florbetaben PET imaging to detect amyloid beta plaques in Alzheimer's disease: phase 3 study. Alzheimers Dement. 2015;11(8):964-74.

3. Curtis C, Gamez JE, Singh U, Sadowsky CH, Villena T, Sabbagh $\mathrm{MN}$, et al. Phase 3 trial of flutemetamol labeled with radioactive fluorine 18 imaging and neuritic plaque density. JAMA Neurol. 2015;72(3):287-94.

4. Yeo JM, Waddell B, Khan Z, Pal S. A systematic review and metaanalysis of (18)F-labeled amyloid imaging in Alzheimer's disease. Alzheimers Dement. 2015;1(1):5-13.

5. Morris E, Chalkidou A, Hammers A, Peacock J, Summers J, Keevil S. Diagnostic accuracy of (18)F amyloid PET tracers for the diagnosis of Alzheimer's disease: a systematic review and meta-analysis. Eur J Nucl Med Mol Imaging. 2016;43(2):374-85.

6. Johnson KA, Minoshima S, Bohnen NI, Donohoe KJ, Foster NL, Herscovitch P, et al. Appropriate use criteria for amyloid PET: a report of the Amyloid Imaging Task Force, the Society of Nuclear Medicine and Molecular Imaging, and the Alzheimer's Association. J Nucl Med. 2013;54(3):476-90.

7. Johnson KA, Minoshima S, Bohnen NI, Donohoe KJ, Foster NL, Herscovitch P, et al. Update on appropriate use criteria for amyloid PET imaging: dementia experts, mild cognitive impairment, and education. J Nucl Med. 2013;54(7):1011-3.

8. Jack Jr CR, Knopman DS, Jagust WJ, Petersen RC, Weiner MW, Aisen PS, et al. Tracking pathophysiological processes in Alzheimer's disease: an updated hypothetical model of dynamic biomarkers. Lancet Neurol. 2013;12(2):207-16.

9. IDEAS study: https://app.ideas-study.org.

10. AMYPAD study: http://www.amypad.eu.

11. Barthel H, Seibyl J, Sabri O. The role of positron emission tomography imaging in understanding Alzheimer's disease. Expert Rev Neurother. 2015;15(4):395-406.

12. Frey KA. Amyloid imaging in dementia: contribution or confusion? J Nucl Med. 2015;56(3):331-2.

13. Kobylecki C, Langheinrich T, Hinz R, Vardy ER, Brown G, Martino ME, et al. 18F-florbetapir PET in patients with frontotemporal dementia and Alzheimer disease. J Nucl Med. 2015;56(3):386-91.

14. Pontecorvo MJ, Arora AK, Devine M, Lu M, Galante N, Siderowf A, et al. Quantitation of PET signal as an adjunct to visual interpretation of florbetapir imaging. Eur J Nucl Med Mol Imaging. 2017. doi:10.1007/s00259-016-3601-4.

15. Seibyl J, Catafau AM, Barthel H, Ishii K, Rowe CC, Leverenz JB, et al. Impact of training method on the robustness of the visual assessment of 18F-Florbetaben PET scans: results from a Phase-3 study. J Nucl Med. 2016;57(6):900-6.

16. Buckley CJ, Sherwin PF, Smith AP, Wolber J, Weick SM, Brooks DJ. Validation of an electronic image reader training programme for interpretation of $[18 \mathrm{~F}]$ flutemetamol $\beta$-amyloid PET brain images. Nucl Med Commun. 2016.

17. Mountz JM, Laymon CM, Cohen AD, Zhang Z, Price JC, Boudhar $\mathrm{S}$, et al. Comparison of qualitative and quantitative imaging characteristics of $[11 \mathrm{C}] \mathrm{PiB}$ and $[18 \mathrm{~F}]$ flutemetamol in normal control and Alzheimer's subjects. Neuroimage Clin. 2015;9:592-8.

18. Schreiber S, Landau SM, Fero A, Schreiber F, Jagust WJ. Alzheimer's disease neuroimaging initiative. comparison of visual and quantitative Florbetapir F 18 positron emission tomography analysis in predicting mild cognitive impairment outcomes. JAMA Neurol. 2015;72(10):1183-90.

19. Thurfjell L, Lilja J, Lundqvist R, Buckley C, Smith A, Vandenberghe R, et al. Automated quantification of $18 \mathrm{~F}$ flutemetamol PET activity for categorizing scans as negative or positive for brain amyloid: concordance with visual image reads. J Nucl Med. 2014;55(10):1623-8.

20. Nayate AP, Dubroff JG, Schmitt JE, Nasrallah I, Kishore R, Mankoff D, et al. Alzheimer's disease neuroimaging initiative. use of standardized uptake value ratios decreases interreader variability of [18F] Florbetapir PET brain scan interpretation. AJNR Am J Neuroradiol. 2015;36(7):1237-44.

21. Hutton C, Declerck J, Mintun MA, Pontecorvo MJ, Devous Sr MD, Joshi AD. Alzheimer's disease neuroimaging initiative. quantification of 18F-florbetapir PET: comparison of two analysis methods. Eur J Nucl Med Mol Imaging. 2015;42(5):725-32.

22. Tuszynski T, Rullmann M, Luthardt J, Butzke D, Tiepolt S, Gertz HJ, et al. Evaluation of software tools for automated identification of neuroanatomical structures in quantitative $\beta$-amyloid PET imaging to diagnose Alzheimer's disease. Eur J Nucl Med Mol Imaging. 2016;43(6): 1077-87.

23. Choi WH, Um YH, Jung WS, Kim SH. Automated quantification of amyloid positron emission tomography: a comparison of PMOD and MIMneuro. Ann Nucl Med. 2016;30(10):682-9.

24. Lilja J, Thurfjell L, Sörensen J. Visualization and quantification of 3-dimensional stereotactic surface projections for $18 \mathrm{~F}$ Flutemetamol PET using variable depth. J Nucl Med. 2016;57(7): 1078-83.

25. Lundqvist R, Lilja J, Thomas BA, Lötjönen J, Villemagne VL, Rowe CC, et al. Implementation and validation of an adaptive template registration method for $18 \mathrm{~F}$-flutemetamol imaging data. J Nucl Med. 2013;54(8):1472-8.

26. Joshi AD, Pontecorvo MJ, Lu M, Skovronsky DM, Mintun MA, Devous Sr MD. A semiautomated method for quantification of F 18 Florbetapir PET images. J Nucl Med. 2015;56(11):1736-41.

27. Nemmi F, Saint-Aubert L, Adel D, Salabert AS, Pariente J, Barbeau $\mathrm{EJ}$, et al. Insight on AV-45 binding in white and grey matter from histogram analysis: a study on early Alzheimer's disease patients and healthy subjects. Eur J Nucl Med Mol Imaging. 2014;41(7): 1408-18.

28. Cattell L, Platsch G, Pfeiffer R, Declerck J, Schnabel JA, Hutton C. Alzheimer's disease neuroimaging initiative. classification of amyloid status using machine learning with histograms of oriented 3D gradients. Neuroimage Clin. 2016;12:990-1003.

29. Heurling K, Buckley C, Vandenberghe R, Laere KV, Lubberink M. Separation of $\beta$-amyloid binding and white matter uptake of $(18) \mathrm{F}$ flutemetamol using spectral analysis. Am J Nucl Med Mol Imag. 2015;5(5):515-26.

30. Chincarini A, Sensi F, Rei L, Bossert I, Morbelli S, Guerra UP, et al. Alzheimer's disease neuroimaging initiative. standardized uptake value ratio-independent evaluation of brain amyloidosis. J Alzheimers Dis. 2016;54(4):1437-57.

31. Vandenberghe R, Nelissen N, Salmon E, Ivanoiu A, Hasselbalch S, Andersen A, et al. Binary classification of ${ }^{18} \mathrm{~F}$-flutemetamol PET using machine learning: comparison with visual reads and structural MRI. Neuroimage. 2013;64:517-25.

32. Obermeyer Z, Emanuel EJ. Predicting the future - big data, machine learning, and clinical medicine. N Engl J Med. 2016;375(13):1216-9.

33. Rullmann M, Dukart J, Hoffmann KT, Luthardt J, Tiepolt S, Patt M, et al. Partial-volume effect correction improves quantitative analysis of $18 \mathrm{~F}$-Florbetaben $\beta$-amyloid PET scans. J Nucl Med. 2016;57(2):198-203. 